\title{
Notiz über das Pankreasnucleoproteid.
}

\author{
Von \\ P. A. Levene und L. B. Stookey. \\ (Aus der physiologisch-chemischen Abteilung des pathologischen Instituts \\ der New-Yorker Staatskrankenhäuser.) \\ (Der Redaktion zugegangen am 21. März 1904.)
}

Die Darstellung einer Nucleinsäure aus dem Pankreasnucleoproteid erwies sich als eine sehr schwierige Aufgabe, deren Lösung nur mit Hilfe des Vèrfahrens von Hammarsten möglich war. Ivar Bang ist es nämlich gelungen, nach dieser Methode sowohl aus dem Pankreasnucleoproteid, als auch direkt aus der Drüse die Guanylsäure zu erhalten. Diese ist eine ganz eigentümliche Nucleinsäure, die sich von den anderen dadurch unterscheidet, daß sie bei der Hydrolyse durch Mineralsäuren kein Adenin und keine Pyrimidinbasen liefert. Der Befund von Bang machte die Vermutung, daß das Pankreasnucleoproteid eine Eiweißverbindung der Guanylsäure sei, sehr wahrscheinlich.

Es ist aber einem von uns gelungen, in der Pankreasdrüse eine Substanz von dor Natur der komplizierteren Nucleinsäuren aufzufinden. Durch diesen Befund ist nun die Natur des Nucleoproteids wieder zweifelhaft geworden, und es schien nötig, festzustellen, ob das Molekül der Substanz auch die kompliziertere Säure enthielt. Da das Nucleoproteid aber eine sehr kleine Ausbeute an der Säure lieferte, so begnügten wir uns damit, die Komponenten derselben direkt aus der Eiweißverbindung zu gewinnen.

Nun hat schon Walter Jones die Anwesenheit von Adenin in Hammarstens Proteid festgestellt. Es war die 
Aufgabe dieser Arbeit, zu entscheiden, ob in diesem Moleküle auch Pyrimidinbasen vorkommen.

Das Nucleoproteid war auf folgende Weise dargestellt. Die frischen in der Hackmaschine verkleinerten Drüsen wurden in eine kochende $0,5 \%$ ige Lösung von Natronkarbonat eingetragen, bis auf $60^{\circ}$ abkühlen gelassen und die Temperatur der Lösung dann schnell durch Hineinbringen von Eis auf $0^{\circ}$ C. herabgesetzt. Diese Vorsicht wurde gebraucht, um die Selbstverdauung möglichst zu verhindern. Die abgekühlte Lösung blieb über Nacht im Eisschrank stehen. Sie wurde dann filtriert und mit Essigsäure versetzt. Es entstand ein Niederschlag, der durch Dekantieren so lange gewaschen wurde, bis die Waschwässer keine Biuretreaktion mehr gaben. Die Substanz wurde dann mit Alkohol und Äther extrahiert, in Vacuo über Schwefelsäure und schließlich im Toluolbad getrocknet und analysiert. Sie enthielt etwa $3 \% \mathrm{P}$.

Die Hauptmenge der Substanz, welche zur Gewinnung der Pyrimidinbasen diente, wurde mit Alkohol weniger ausgiebig extrahiert.

Der noch feuchte Niederschlag wurde mit $25 \%$ iger Schwefelsäure im Autoklaven drei Stunden auf $175^{\circ}$ erhitzt. Aus der vom Melanin abfiltrierten Flüssigkeit wurde die Schwefelsäure in üblicher Weise entfernt, und dann nach Kossels Verfahren die Pyrimidinbasen, das Arginin und das Histidin mit Silbernitrat uud Baryt niedergeschlagen. Zur Trennung der Basen befreiten wir den Niederschlag von Silber und von dem anhaftenden Baryt und konzentrierten die dabei erhaltene Lösung bei stark vermindertem Druck. Der Rückstand zeigte zunächst keine große Neigung zur Kristallisation; erst als wir ihn in 10\% oiger Schwefelsäure lösten, bildeten sich über Nacht Kristalle, die das typische Aussehen des Thymins besaßen.

Die Menge reichte aber zur Analyse nicht aus. Die Mutterlauge wurde dann in Vacuo über Schwefelsäure stehen gelassen, dabei entstand eine zweite Ausscheidung, die dem Uracil ähnlich sah. Die zwei Niederschläge wurden dann vereinigt, aus 2\% iger Schwefelsäure umkristallisiert, mit Alkohol und Äther gewaschen und zur Analyse verwandt. Die Substanz, 
obwohl aus Schwefelsäure umkristallisiert, hielt keine Spur von der Säure zurück und gab mit Pikrinsäure keinen Niederschlag. Die Analyse ergab die folgenden Zahlen:

$0,1375 \mathrm{~g}$ der Substanz gaben $29,5 \mathrm{ccm}$ Stickstoff (über $50 \%$ iger Kalilauge) bei $750 \mathrm{~mm}$ Bar. und $23^{\circ} \mathrm{C}$. T.

Berechnet für $\mathrm{C}_{5} \mathrm{H}_{6} \mathrm{~N}_{2} \mathrm{O}_{2}$ für $\mathrm{C}_{4} \mathrm{H}_{4} \mathrm{~N}_{2} \mathrm{O}_{2}$ Gefunden

$$
\mathrm{N}=22,22 \% \quad 25,05 \% \quad 24,40 \%
$$

Die Substanz war also ein Gemisch von Thymin und Uracil.

Man wird also annehmen müssen, daß auch das Pankreasnucleoproteid ein Derivat einer komplizierten Nucleinsäure ist.

\section{Literatur :}

Bang, Diese Zeitschrift, Bd. XXVI, S. 133; Bd. XXXI, S. 411.

W. Jones und Whipple, Amer. Journ. of Physiol., v. 7.

Levene, Diese Zeitschr., Bd. XXXII, XXXVII, XXXVIII.

\section{Berichtigung. \\ Von \\ E. Schulze.}

In einer von mir in Verbindung mit N. Cast or o in Bd. XXXVIII dieser Zeitschrift publizierten Abhandlung ist auf Seite 204 in der zweiten Anmerkung bei Besprechung der Bildung von Homogentisinsäure aus Tyrosin gesagt worden, daß nach F. Mittelbach die Eingabe von Tyrosin nicht ein Ansteigen der Homogentisinsäure im Harn in dem von Wolkow und Baumann beobachteten Umfange bewirkt habe. Wir verwiesen dabei auf das Jahrbuch der Chemie von R. Meyer, Jahrgang XI (1901), in welchem es auf S. 235 heißt: «Doch findet Fr. Mittelbach, daß die Gegenwart der Harnsäure hierbei (sc. bei Bestimmung der Homogentisinsăure im Harn) im Mittel einen Fehler von 6,1\% bedingt. ${ }^{1}$ ) Ferner bewirkte die Eingabe von Tyrosin nicht ein Ansteigen der Homogentisinsäure in dem von Wolkow und Baumann beobachteten Umfange.» Nun hatte aber Herr Professor Huppert die Gefälligkeit, mich darauf aufmerksam zu machen, daß in den Versuchen Mittelbachs jene Erscheinung nur in einem Falle, bei Verabreichung von einer relativ großen Tyrosinmenge, eingetreten ist, während dagegen bei Verabreichung geringerer Dosen das Tyrosin im Harn als Homogentisinsäure vollständig wieder zum Vorschein kam. Die obige Angabe bedarf also einer Richtigstellung, bezw. einer Ergänzung.

1) Archiv für klin. Medizin, Bd. 71, S. 50. 